\title{
Adaptation and research development in the spectrum of methods with advanced access in the area of communication and computer system
}

\author{
Nderim Zeqiri \\ State University in Tetova - Faculty of Applied Sciences (FSHZ)-Mechatronics, 1200-Tetovo, R. Macedonia \\ Email: nderimzeqiri@gmail.com
}

\begin{abstract}
In some models that set new principles of work, there appears to require the implementation and adaptation of computer resources in terms of hardware and software adaptation. This method entails the extension of the current system versus research perspectives in the context of programs, models and restructuring in line with other development, hardware or software, or adjustment to learning in electronic form; the interaction of many processes, to create a sustainable system through increased research and adoption of safety and suitability. So in this paper we present models and alternative approaches to achieve a better perspective in the field of restructuring and adjustment of the model with the program and hardware resources. In many cases is needed a quick access method which is specified in the context of generating appropriate values for digital systems
\end{abstract}

Keywords: Adaptation, advanced access, communications, computer, system

\section{Introduction}

In some cases, the workflow management of computer systems in different institutions needs the introduction of methods which provide a better performance of the work. Above all, to achieve this, should collect data about the computer system and identification of certain errors. To realize a complete approach regarding the structuring of the system needs to find methods that are adaptive in terms of different spectrum of tools that are in use. Oftentimes certain errors occur during selection of the method of working, creating unnecessary extra work on certain structuring [1],[2]. This extra work creates other costs, and other investments in the context of adapting general structure of computer system, for implementation of various tasks, such as: maintenance of the system, the creation of programs applicable to certain implementations, documenting programs creation of input-output resources, and many other segments and create a grading system that is sustainable and practical implementation in term [3].

One of the most persistent and critical issues plaguing development in general, and the use of technology in education specifically, is equity.

Differences among people and societies continue to grow, affecting not only economic disparities but also health, environmental, educational, and information disparities. The challenge for many governments and organizations continues to be the need to devise better ways to bridge this gap. It is clear that education, in all sectors, is the single most important means. Development projects and reform efforts are not institutionalized automatically or easy to sustain.

Achieving this requires active strategies and policies to ensure that initial investments in infrastructure, technology, training, and integrating the use of computers in teaching and learning are sustained and encouraged to grow. This will not occur without sufficient support from essential staff, especially principals and teachers. One of the most important strategies to sustain and expand projects is to involve teachers and principals, as well as members of the community from the very beginning in all decision-making and implementation processes. A continuous effort is needed to engage staff in meaningful planning activities, including: Carrying out and interpreting assessments; Planning and designing the system;

- determining how the computers will be used

- to support teaching and learning;

- choosing software; 
- deciding how professional development and

- training activities will be managed;

- determining how computer use will be allocated among teachers and students and across grades and subjects;

- devising monitoring and evaluation processes; and

- Deciding how the school budget may need to be modified to expand and support the system.

The more meaningful and authentic teacher and principal involvement is in planning and managing a project, the greater the chance that the school and staff will take ownership of the system and apply the time and resources needed to ensure that it grows[2],[3].

\section{Advanced approach to system objects}

One approach is advanced in terms of management and the creation of the system if the system is operational, no unnecessary interruptions and user possesses all the tools to function automatically. To achieve this of course, must be projected flow of information scheme to achieve certain objectives [5].

Scheme implemented in Fig. 1, shows the main blocks of information flow in a direction designed by programmers and operators.

To follow up on late work requirements, must consult with professional people who know the technology work, or function to which the requirements of specific application program [7].

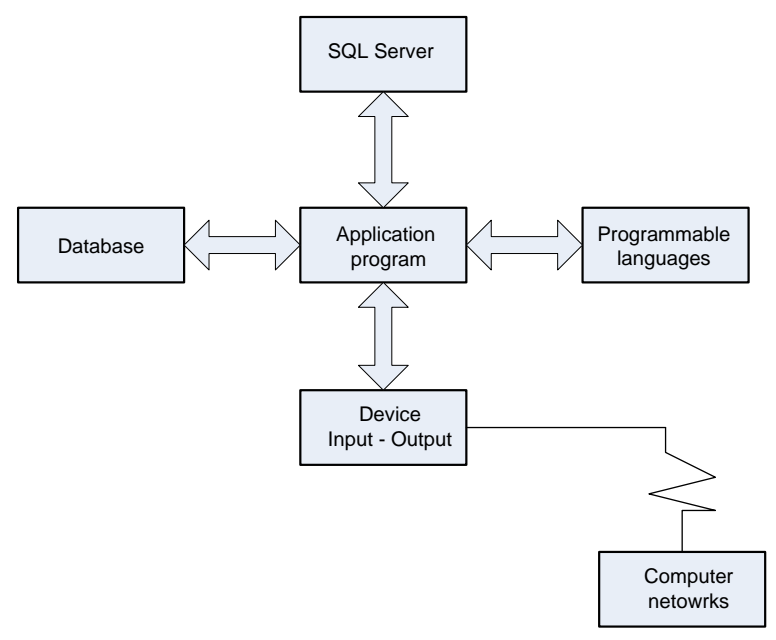

Fig.1: This scheme is the description of information flow, across different modules

\section{Calculation of the system performance}

The performance can be calculated by certain formulas. For example, Px-is performance of a first computer, and Pyperformance of a second computer. Time of completion of relevant works, noted as Tx and Ty [8][9]. Where Tx and Ty are the execution time of Px and Py computers.

Table.1: System performance

\begin{tabular}{cccc}
\hline Tasks & Px & Py \\
\hline P1 (sec) & 1 & 1.5 \\
P2 (sec) & 2 & 1.5 \\
P3 (sec) & 1.7 & 1.8 \\
P4 (sec) & 1.9 & 2.1 \\
P6 (sec) & 2.2 & 2.0 \\
P7 (sec) & 3 & 3.3 \\
\hline
\end{tabular}


To calculate the average time (in second) is needed the following formula:

$P_{x}=\frac{1+2+1.7+1.9+2.2+3}{7}=1$

$\mathrm{P}_{\mathrm{x}}=\frac{1.5+1.5+1.8+2.1+2.0+2.8}{7}=1.74$

$\mathrm{T}_{\mathrm{x}}=\frac{1}{\mathrm{P}_{\mathrm{x}}}=\frac{1}{1.68}=0.595$

$\mathrm{T}_{\mathrm{y}}=\frac{1}{\mathrm{P}_{\mathrm{y}}}=\frac{1}{1.74}=0.574$

$\mathrm{T}_{\mathrm{x}}=0.595>\mathrm{T}_{\mathrm{y}}=0.574$

With better performance is Tx, because:

$\mathrm{Tx}>\mathrm{Ty}$

Program code for processing and recycling respective cycles on the basis of the respective vectors elements:

\#include <iostream>

\#include <Windows.h $>/ /$ to use sleep () command

using namespace std;

int main ()

\{

int $\mathrm{n}, \mathrm{i}, \mathrm{j}, \mathrm{B}[8]=\{2,4,16,32,64,128,256,512\}$;

float $\mathrm{m}$;//to uses rational number

cout $<<$ "\n The delay time is!"<<endl;

$\operatorname{cin}>>\mathrm{m}$;

cout $<<" \mathrm{~m}=$ " $<<\mathrm{m}<<"$ sec $"<<$ endl;

cout $<<"$ "In how many time do you want to be repeated this vector"<<endl;

cin $>>n$;

cout $<<$ "n $=$ "<<n $<<$ " n-times " $<<$ endl;

\{

for $(j=1 ; j<=m ; j++)$

\{

for $(i=0 ; i<=7 ; i++)$

\{

cout $<<" \ln \mathrm{B}["<<\mathrm{i}+1<<"]="<<\mathrm{B}[\mathrm{i}]<<$ endl $<<$ endl;

\}

cout<<"---------------------------------------------------"<<endl;//

sleep (m*1000);

\}

\}

return 0 ; 
The aim of this work is the way of quickly accessing the system, replacement of certain modules if they are obsolete, or are not adaptive to new resources. In fact, this also relates to the subject which deals with software reuse. Since the focus of the scheme have set application program, so important point here as we have taken the management of computer resources and software [10]. The program must be installed on the computer to perform certain functions, while the computer can become part of the computer network, as shown in Fig.1.

Ordering the elements in the computer system where the program is applicable is very important work which should, be planned in advance. There are many parameters which need to be cautious in certain adaptations. The purpose of this paper is to prove that not only the speed, capacity, and power of the computer affects the performance of the implementation of various works, but also affects the update and good planning in the context of adaptation to work, according to a block-scheme, or specific algorithms, Fig.2.

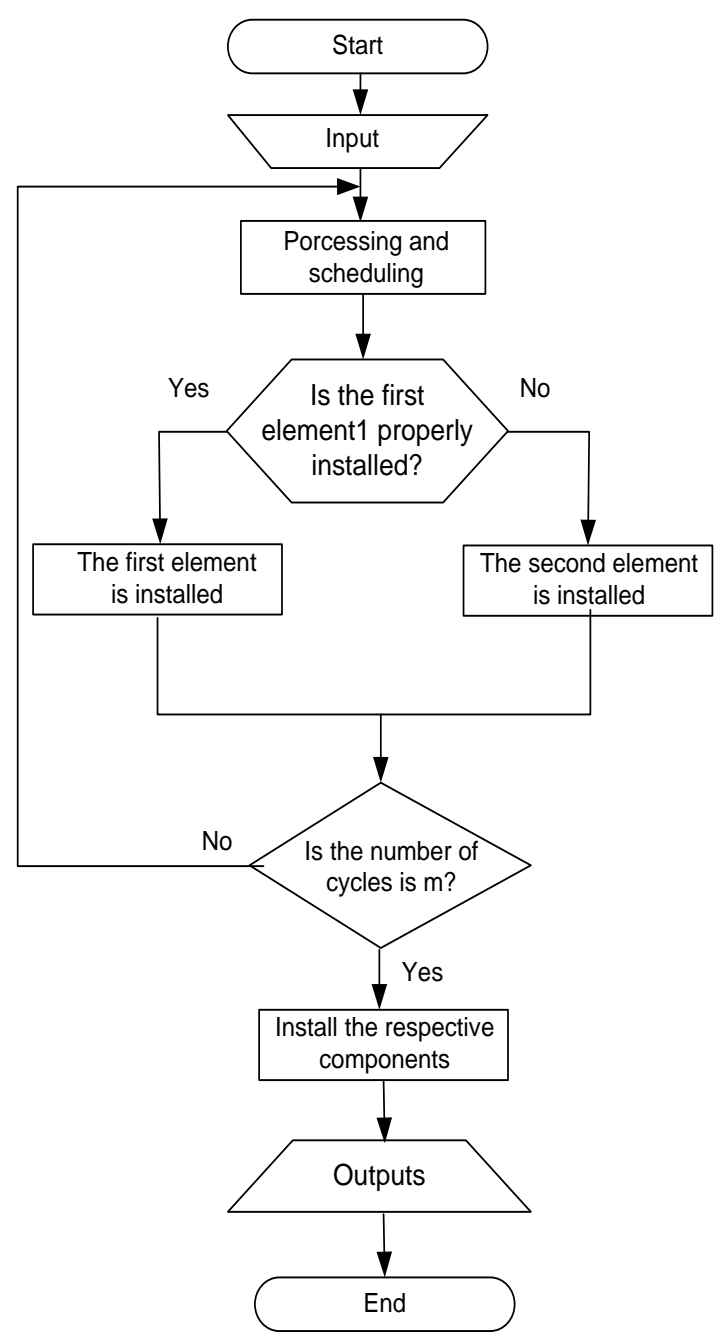

Fig.2: The block-diagram for implementation of adaptation and replacement of elements of computer system

This method entails the extension of the current system versus research perspectives in the context of programs, models and restructuring in line with other development, hardware or software, or adjustment to learning in electronic form. To realize a complete approach regarding the structuring of the system needs to find methods that are adaptive in terms of different spectrum of tools that are in use. Here is the relevant software module created for sustainable access. At first glance it seems that this program and this algorithm is very flexible, and extremely convenient for different systems, because access is direct. Requires only a modest knowledge of access to certain elements in the organized structure, and since we are dealing with a one-dimensional field and appropriate. 


\section{Determining the factious values during the recycling in the area of communications}

Sometimes it is very difficult to find numerical values appropriate for efficient communication in a certain channel. But, using the programming this activity can simplify the procedure of creating adequate access to various systems. In the context of command, this procedure creates the appropriate channel for the movement of information, because the movement of data referred to the program and the interface as a media liaison.

Generating appropriate values with digital-analog nature, and receipt of information and their conversion, using the program simplifies the work. Because there is no need for additional modules, and procedures, command and conversion modules would be somewhat more complex.

Program code for processing and recycling respective cycles on the basis of the respective vectors elements, where we can simulate any communications, such as a :

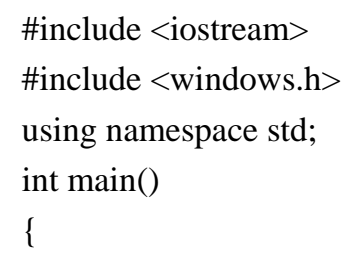

Output results,

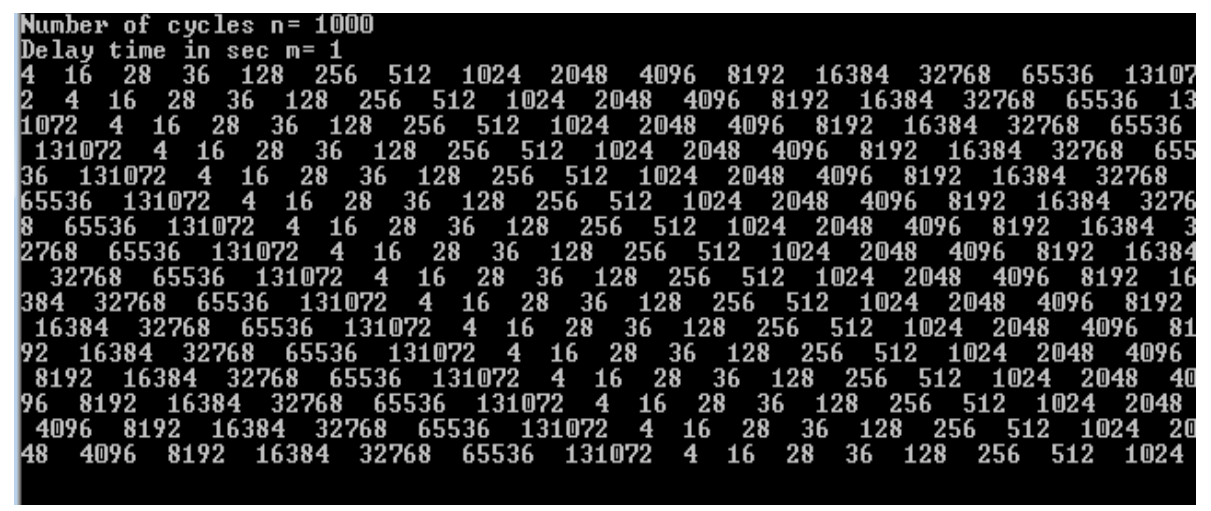

Fig.3: Output results from above program. 
Table. 2

\begin{tabular}{|l|c|c|c|c|c|}
\hline \multicolumn{7}{|c|}{ Decimal number and their binary equivalent } \\
\hline decimal equivalent & $\ldots$ & 64 & 128 & 256 & $\ldots$ \\
\hline delay time (millisecond) & $\ldots$ & 10 & 10 & 10 & $\ldots$ \\
\hline Binary equivalent numbers & $\ldots$ & 1000000 & 10000000 & 10000000 & $\ldots$ \\
\hline
\end{tabular}

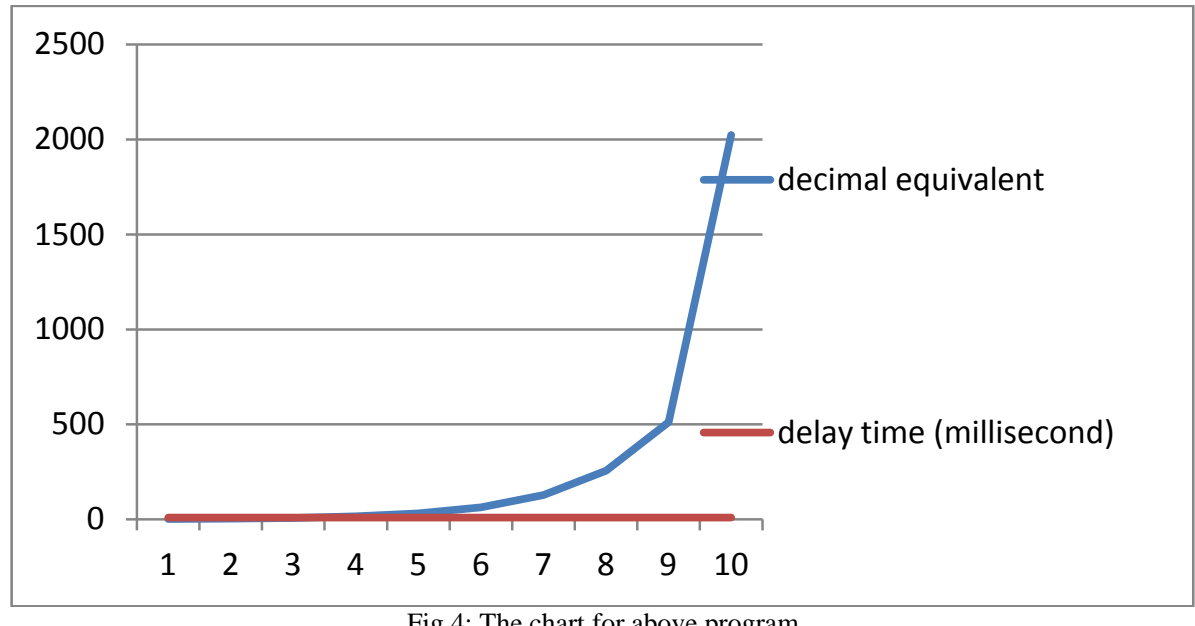

Fig.4: The chart for above program

We can realize the respective algorithms, for any communications, according to a block-scheme, Fig 3 . With the above program not only can achieve the appropriate simulation, but this program can be expanded and implemented as a successful communication with the relevant computer ports. Through computer ports, the interface may be implementing various communications, generating foreign values, values that have digital nature. These digital values are of type, $4,8,16,32,64,256,1024$, etc (their binary equivalent). These values are appropriate and within a certain communication.

Why this communication is flexible, visible and clear? Looking at Fig.3, we can conclude that such values can be generated in different channels, previously defined. These values may be stochastic nature, but we identify in advance those values. [5][6]

These values are determined in the context of the array and algorithm. So these fictitious values, defined by the programmer, they create the possibility of establishing certain channels and more flexible. The possibility of updating of these values and the establishment of communication, facilitates the dimensioning of communication, but also broadens the horizon of practical implementation of this system.

\section{Initialize the interface for transfer of information and communications}

During initialization of the interface, I/O routine has continued control of this situation. Thus the status of the device can be tested (established control loops), this is because the transfer of information carried in an appropriate manner, and so successful. The ways of testing and defining the direction for the implementation of the transfer, when the device is ready, allows access to be adequate and the management time of system resources within communication channel to be the optimal (or delay time with reasonable length) [7][8][9].The general form of presentation with the following algorithm can be used for special occasions, when events are defined in advance, according to a certain order, in accordance with its technological and system requirements. These values are fictitious because they can be easily modified and adapted. Adaptation is about the ways of communication channels in certain computer ports, and connection of external equipment. Following algorithm, the blocks of the condition, always sets the question, whether the devices are ready to work, because so values initiated by the program, and initiated interface when transmitting information in real time. 


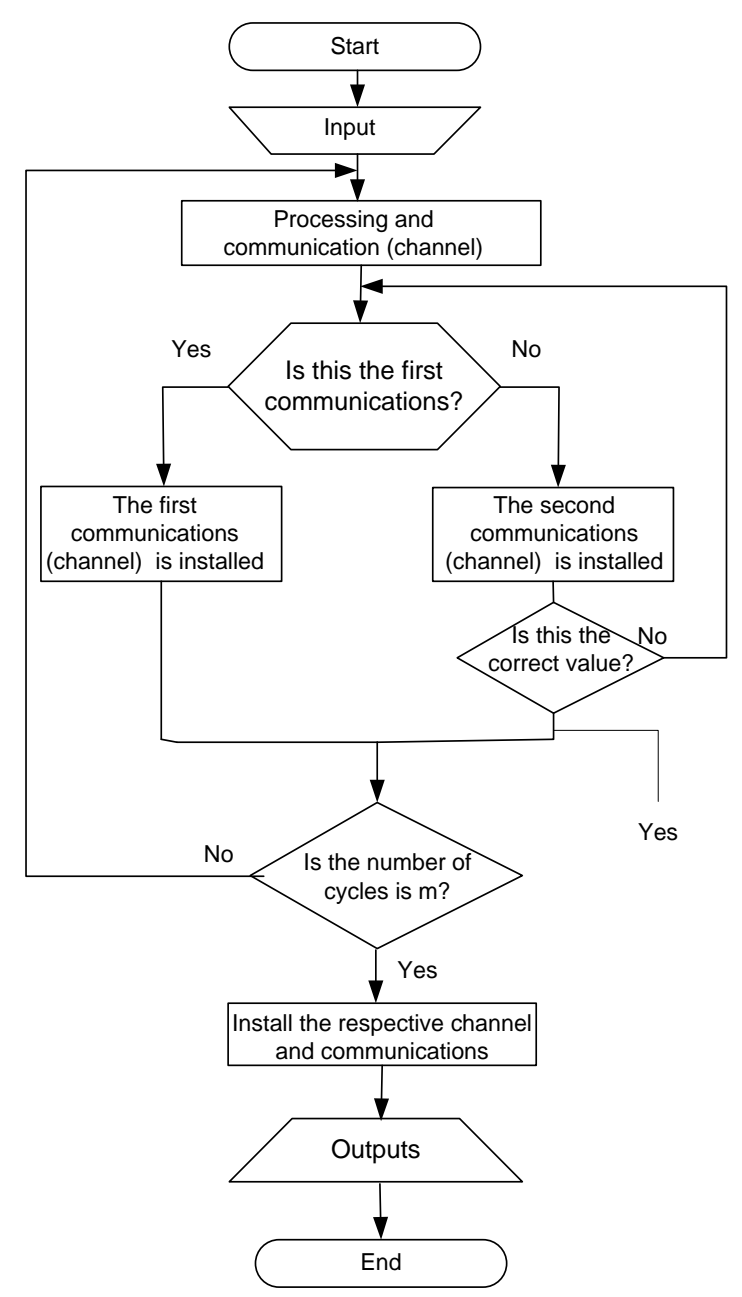

Fig.5: The block-diagram for implementation of respective channel communications

For example: if there needs to be supplied an LED diode which is located within an interface, if it should be initiated every $10 \mathrm{~ms}$ and other LED diodes, in consecutive order, then, the activity of the program should initiate the LED controller, namely telecommunications networks channel, and sets the delay time of $10 \mathrm{~ms}$ (millisecond). Delay time is realized with the program, most commonly in the form of loops, as the algorithm in Fig. 4. The delay time program used in this case, indicate/used as a synchronization of deterministic events.

Program I/O is the best method of transmission, especially for the realization of other activities, especially when the hardware interface between I/O devices and the computer must be simple and practical.

\section{Conclusion}

In this paper, is presented and realized a respective method, a method for finding appropriate values for fictitious and deterministic values and transmission of information in certain channels. The flexibility of the program enables easy updating, to create a new approach in the communication system. These values can always be deterministic for external interface, and always can be used as a certain generation of values, according to a specific domain. This method entails the extension of the current system versus research perspectives in the context of programs, models and restructuring in line with other development, hardware or software, or adjustment to learning in electronic form. These values are fictitious because they can be easily modified and adapted.

Adaptation is about the ways of communication channels in certain computer ports, and connection of external equipment. By using this system facilitated the management of channels communications. At first glance it seems that this program and this algorithm are very flexible, and extremely convenient for different systems. I to make a good system that must be a functional and well structured system require basic knowledge in relevant programming languages and relevant communications systems. In this paper are presented the method to determine fictitious values 
during the recycling in the area of communications and computer systems with advanced access, and updating the values. Obviously, the incorporation of certain sequences within certain levels provides flexible access to certain parameters [1].

One of the best options is access and to familiarity with fictitious values which are close at any time, and to update options to certain values all of those things is to achieve a certain digital controls. In telecommunication systems is very important a delay time, sometimes this value should be very small, or indistinct, but sometimes this delay time can be fictitious for certain destinations in the acceptance and delivery of certain values.

Many communication systems are based on the algorithm that allows special access. This approach has set the path for the realization of rapid communication and "error-free". Although errors can occur any time, always preferred to create more secure communications and sophisticated. In this paper is presented a restructuring under a certain model and was given advanced programming approach in terms of creating the model and the possibility of expanding the scope and implementation. To make this clearer the results are given and are explained aspects of their priority due to highlight the effects and put comparative values in terms of their development.[2][3].

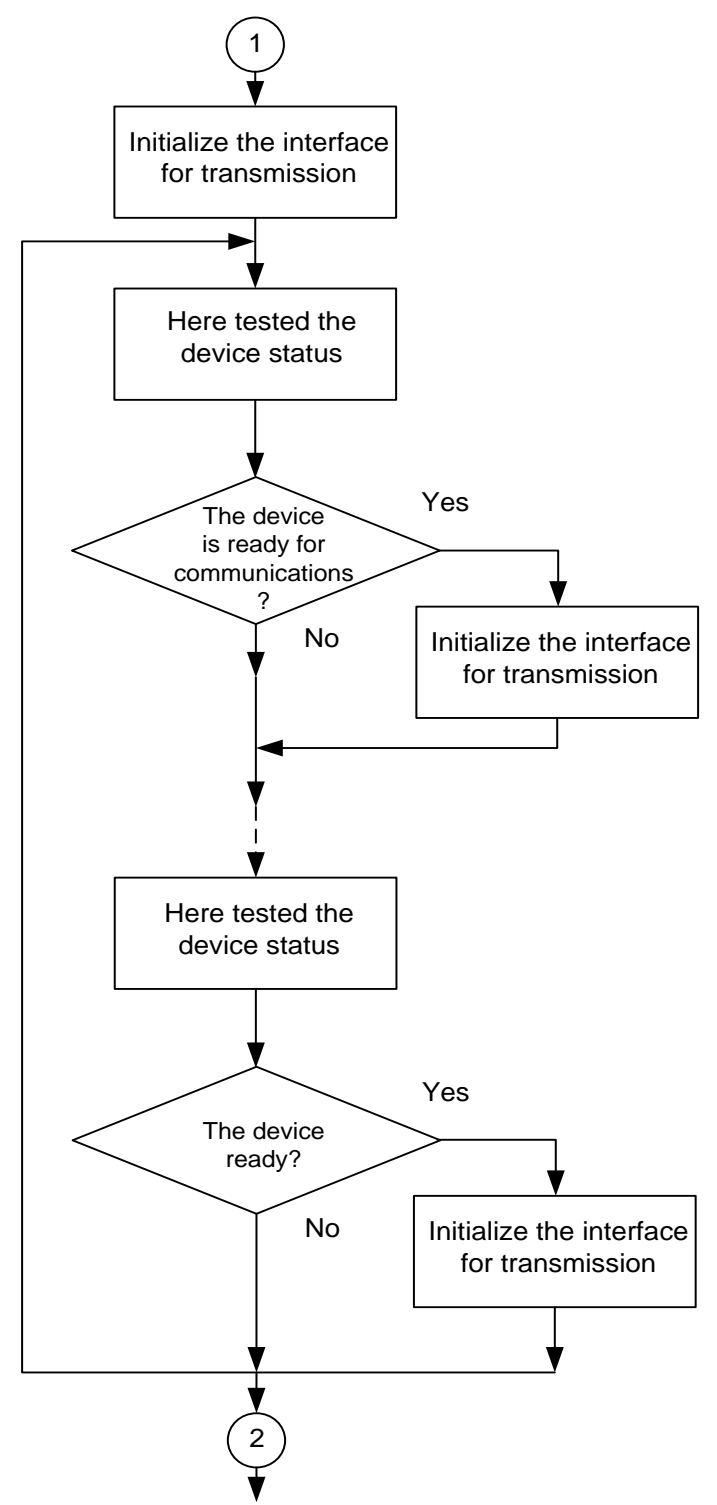

Fig.6: The part of block diagram to initialize the interface for transmission 


\section{References}

[1] Trading Bit, Message, and Time Complexity of Distributed Algorithms Johannes Schneider and Roger Wattenhofer. 25th International Symposium on Distributed Computing (DISC), Rome, Italy, September 2011.

[2] D. Burger and T. Austin, "The SimpleScalar Tool Set Version 2.0," Univ.of Wisconsin, Madison, Tech. Rep. CS-TR-97-1342, 1997.

[3] M. Rosenblum, E. Bugnion, S. Devine, and S. A. Herrod, "Using the SimOS machine simulator to study complex computer systems," ACM Trans. Modeling Computer Simulation, vol. 7, no. 1, pp. 78-103.

[4] R. N. Ibbett, "Hase DLX simulator,” IEEE Micro, vol. 20, no. 3, pp. 57-65, May/Jun. 2000.

[5] J. L. Bechennec, "ASF: A teaching and research object-oriented simulation tool for computer architecture design and performance evolution," in Proc.Workshop Computer Architecture Education (WCAE-98), Barcelona, Spain, Jun. 27, 1998, pp. 93-96.

[6] Potashnik, Michael, and Adkins, Douglas. Cost Analysis of Information Technology Projects in Education: Experiences from Developing Countries. Education and Technology Series, vol. 1, no. 3. Washington:World Bank, 1996.

[7] Stephan Holzer, Yvonne Anne Pignolet, Jasmin Smula, and Roger Wattenhofer. 6th International Workshop on Algorithms for Sensor Systems (ALGOSENSORS), Wireless Ad Hoc Networks and Autonomous Mobile Entities, Bordeaux, France, July 2010.

[8] D. Burger and T. Austin, "The SimpleScalar Tool Set Version 2.0," Univ.of Wisconsin, Madison, Tech. Rep. CS-TR-97-1342, 1997.

[9] M. Rosenblum, E. Bugnion, S. Devine, and S. A. Herrod, "Using the SimOS machine simulator to study complex computer systems," ACM Trans. Modeling Computer Simulation, vol. 7, no. 1, pp. 78-103.

[10] Y. Zhang and G. B. Adams, “An interactive, visual simulator for the DLX pipeline,” IEEE TCCA Newslett., pp. 9-12, Sep. 1997.

[11] R. N. Ibbett, "Hase DLX simulator,” IEEE Micro, vol. 20, no. 3, pp. 57-65, May/Jun. 2000.

[12] J. L. Bechennec, "ASF: A teaching and research object-oriented simulation tool for computer architecture design and performance evolution," in Proc.Workshop Computer Architecture Education (WCAE-98), Barcelona, Spain, Jun. 27, 1998, pp. 93-96.

[13] Potashnik, Michael, and Adkins, Douglas. Cost Analysis of Information Technology Projects in Education: Experiences from Developing Countries. Education and Technology Series, vol. 1, no. 3. Washington:World Bank, 1996.

[14] Romiszowski, Alexander."New Technologies for Professional Education, Training, and Human Resource Development." In Claudio de Moura Castro (ed.). Education in the Information Age, Washington: Inter-American Development Bank, 1998.

[15] Rudenstine, Neil L. "The Internet and Education: A Close Fit." The Chronicle of Higher Education (February 21, 1997).

[16] Rusten, E., Contreras-Budge, E., and Tolentino, D. Enlaces: Building a National Learning Network, LearnLink Case Study Summary.Washington: Academy for Educational Development, 1999. 